Supplementary Information for

\title{
Impact of phyllosilicates on amino acid formation under asteroidal conditions
}

V. Vinogradoff ${ }^{1,2 *}$, L. Remusat ${ }^{2}$, H.L. McLain ${ }^{3,4}$, J.C. Aponte ${ }^{3,4}$, S. Bernard ${ }^{2}$, G. Danger ${ }^{1,5}$, J.P. Dworkin $^{3}$, J.E Elsila ${ }^{3}$, and M. Jaber ${ }^{5,6}$

${ }^{1}$ Physique des Interactions Ioniques et Moléculaires, PIIM UMR-CNRS 7345, Aix-Marseille Université, 13397-Marseille, France

${ }^{2}$ Muséum National d’Histoire Naturelle, Sorbonne Université, UMR CNRS 7590, Institut de minéralogie, de physique des matériaux et de cosmochimie, Paris, France.

${ }^{3}$ Solar System Exploration Division, NASA Goddard Space Flight Center, Greenbelt, Maryland 20771, United States

${ }^{4}$ Department of Chemistry, The Catholic University of America, Washington, DC 20064, United States

${ }^{5}$ Institut Universitaire de France (IUF)

${ }^{6}$ Sorbonne Université, CNRS UMR 8220, LAMS, case courrier 225, 4 pl. Jussieu 75252 Paris cedex 05, France

* Corresponding author: Vassilissa Vinogradoff

Email: vassilissa.vinogradoff@univ-amu.fr 
Supplementary Methods for smectite characterization.

X-ray diffraction analysis

The XRD patterns were collected at the X-ray diffraction facility of IMPMC (Paris, France). The X-ray powder diffraction patterns were recorded using an X'Pert Pro Panalytical diffractometer equipped with a $\mathrm{CoK} \alpha_{1,2}$ radiation source $(\lambda \mathrm{K} \alpha 1=1.78897 \AA, \lambda \mathrm{K} \alpha 2=1.79285 \AA)$ and an X’Celerator detector, in Bragg Brentano geometry $\left(0.04^{\circ}\right.$ Soller slits, $0.5^{\circ}$ programmable divergence slit, $1^{\circ}$ incident antiscatter slit, and $0.5^{\circ}$ diffracted antiscatter slit). The XRD patterns were collected between 3 and $60^{\circ} 2 \theta$ with a step size of $0.0167^{\circ} 2 \theta$ and a counting time per step of 6.7 s. Less than $0.5 \mathrm{mg}$ of grounded samples were placed on a Si pellet and introduced in the diffractometer. The analysis on the pellet may have influenced the profile of diffraction pattern due to random geometry of the phyllosilicate.

\section{Infrared spectroscopy analysis}

FTIR analyses were performed using a Vertex 70 spectrometer (Bruker) at the Musée de l'Homme (Paris, France). Around 0.5 mg of powdered sample was pressed onto a diamond crystal surface using a single reflection in the attenuated total resonance device, ATR-quest (Specac). The spectrum was acquired from 370 to $4000 \mathrm{~cm}^{-1}$ with a spectral resolution of $4 \mathrm{~cm}^{-1}$. Ninety-six spectra were accumulated (vs. 128 for the background). Each IR spectrum was corrected with a linear elastic concave baseline and normalized to its total absorbance area (using Bruker Opus software). 

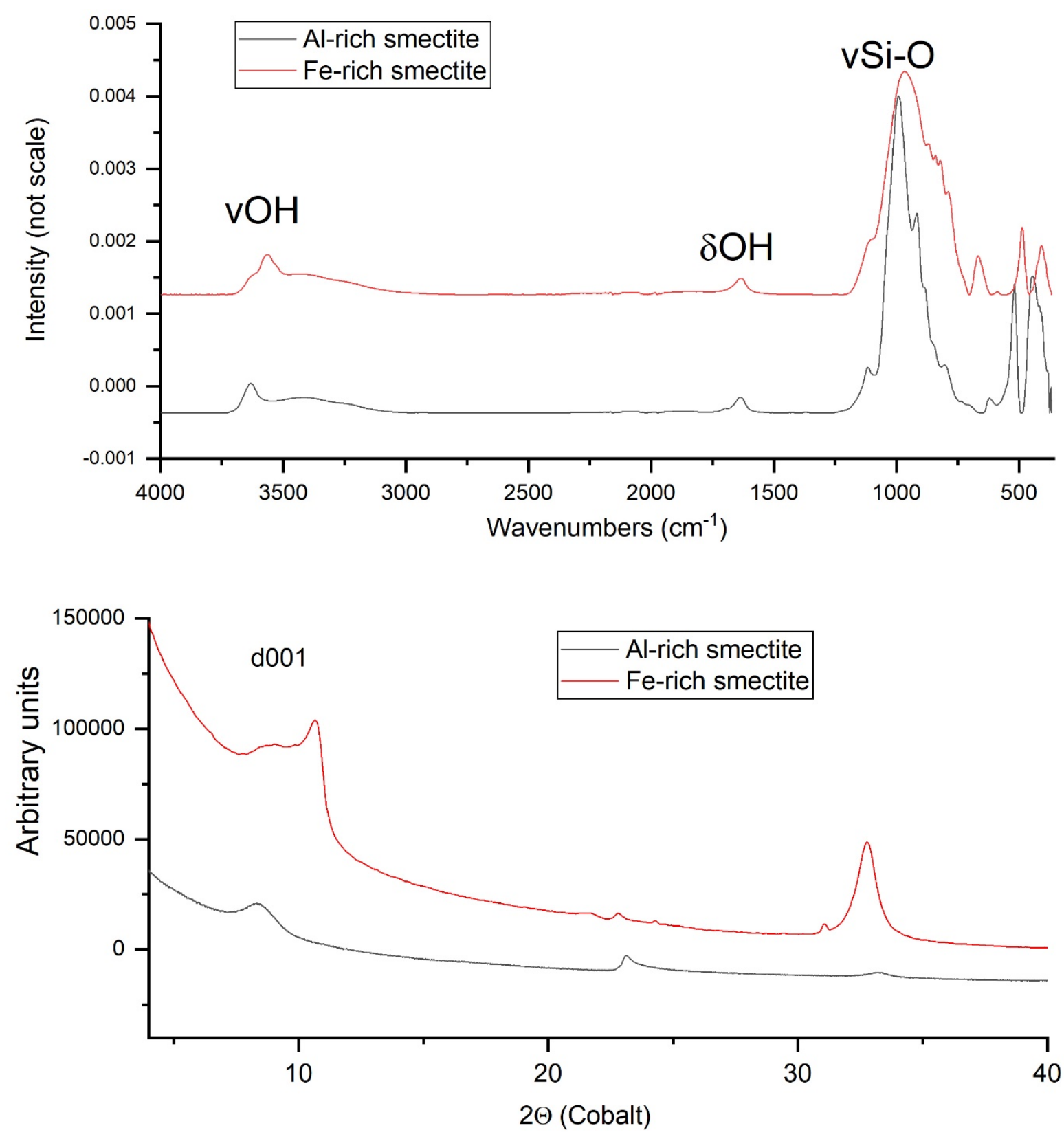

Figure SI1 Infrared spectra and X-ray diffraction spectra of the synthetic smectites used in this study. Main IR band are indicated ( $\mathrm{vOH}$ : stretching $\mathrm{OH}$ from smectite, $\delta \mathrm{OH}$ stretching from adsorbed water, vSi-O, stretching Si-O from smectite). On the XRD spectra is indicated the location of the d001 diffraction band corresponding to the interlayer space of smectites. 
Table SI1. Abundance of amino acid analyzed in the hydrothermal samples at 0, 2, 7 and 31 days (values are reported in $\mu \mathrm{M}$ with standard deviation errors over 3 analyses, see methods).

\begin{tabular}{|c|c|c|c|c|c|c|c|c|c|}
\hline Exp. & Glycine & $\alpha$-Alanine & $\beta$-Ala & $\gamma$-ABA & $\beta-\mathrm{ABA}$ & $\beta$-AIB & $\alpha-\mathrm{ABA}$ & $\alpha$-AIB & $\begin{array}{l}\text { Total } \\
\text { amino } \\
\text { acids }\end{array}$ \\
\hline \multicolumn{10}{|l|}{ HMT } \\
\hline 0 day & $0.42 \pm 0.10$ & $<0.1$ & $<0.1$ & $<0.1$ & $<0.1$ & $<0.1$ & $<0.1$ & $<0.1$ & $<0.5$ \\
\hline 2 days & $7.67 \pm 0.25$ & $<0.1$ & $0.96 \pm 0.03$ & $<0.1$ & $<0.1$ & $<0.1$ & $<0.1$ & $<0.1$ & 9 \\
\hline 7 days & $190.64 \pm 5.06$ & $6.97 \pm 0.86$ & $7.91 \pm 0.20$ & $1.17 \pm 0.24$ & $0.59 \pm 0.03$ & $0.89 \pm 0.19$ & $<0.1$ & $<0.1$ & 208 \\
\hline 31 days & $573.44 \pm 15.15$ & $30.31 \pm 2.11$ & $15.35 \pm 0.38$ & $11.01 \pm 2.88$ & $1.22 \pm 1.05$ & $2.39 \pm 0.77$ & $1.22 \pm 0.27$ & $0.30 \pm 0.23$ & 635 \\
\hline \multicolumn{10}{|c|}{ HMT+CA } \\
\hline 0 day & $20.90 \pm 1.06$ & $<0.1$ & $<0.1$ & $<0.1$ & $<0.1$ & $<0.1$ & $<0.1$ & $<0.1$ & 21 \\
\hline 2 days & $53.43 \pm 1.45$ & $0.75 \pm 0.10$ & $2.15 \pm 0.14$ & $<0.1$ & $<0.1$ & $<0.1$ & $<0.1$ & $<0.1$ & 57 \\
\hline 7 days & $399.59 \pm 8.67$ & $8.59 \pm 0.63$ & $14.96 \pm 0.96$ & $1.45 \pm 0.26$ & $0.66 \pm 0.29$ & $1.20 \pm 0.25$ & $1.19 \pm 0.05$ & $<0.1$ & 428 \\
\hline
\end{tabular}




\begin{tabular}{|c|c|c|c|c|c|c|c|c|c|}
\hline 31 days & $396.82 \pm 7.66$ & $15.35 \pm 1.46$ & $18.22 \pm 1.21$ & $1.53 \pm 0.25$ & $0.71 \pm 0.02$ & $1.37 \pm 0.31$ & $1.18 \pm 0.04$ & $0.23 \pm 0.12$ & 436 \\
\hline $\begin{array}{l}\mathrm{HMT}+\mathrm{A} \\
\text { rich smecti }\end{array}$ & & & & & & & & & \\
\hline 0 day & $1.56 \pm 0.12$ & $0.20 \pm 0.02$ & $<0.1$ & $<0.1$ & $<0.1$ & $<0.1$ & $<0.1$ & $<0.1$ & 2 \\
\hline 2 days & $96.98 \pm 4.61$ & $0.85 \pm 0.07$ & $4.38 \pm 0.34$ & $0.89 \pm 0.07$ & $<0.1$ & $<0.1$ & $<0.1$ & $<0.1$ & 103 \\
\hline 7 days & $149.57 \pm 3.73$ & $1.91 \pm 0.15$ & $6.04 \pm 0.43$ & $0.92 \pm 0.14$ & $0.59 \pm 0.02$ & $0.77 \pm 0.08$ & $<0.1$ & $<0.1$ & 160 \\
\hline 31 days & $1314.34 \pm 16.90$ & $57.40 \pm 2.58$ & $17.81 \pm 0.69$ & $73.01 \pm 5.56$ & $3.82 \pm 3.09$ & $2.05 \pm 1.33$ & $1.27 \pm 0.07$ & $0.25 \pm 0.17$ & 1470 \\
\hline $\begin{array}{l}\mathrm{HMT}+\mathrm{F} \\
\text { rich smecti }\end{array}$ & & & & & & & & & \\
\hline 0 day & $0.79 \pm 0.06$ & $0.44 \pm 0.04$ & $<0.1$ & $<0.1$ & $<0.1$ & $<0.1$ & $<0.1$ & $<0.1$ & 1 \\
\hline 2 days & $45.44 \pm 0.77$ & $0.73 \pm 0.05$ & $1.62 \pm 0.81$ & $<0.1$ & $<0.1$ & $<0.1$ & $<0.1$ & $<0.1$ & 48 \\
\hline 7 days & $25.36 \pm 0.48$ & $1.01 \pm 0.05$ & $3.91 \pm 0.24$ & $0.89 \pm 0.01$ & $<0.1$ & $<0.1$ & $<0.1$ & $<0.1$ & 31 \\
\hline 31 days & $223.11 \pm 5.70$ & $10.39 \pm 0.82$ & $11.89 \pm 0.90$ & $20.13 \pm 8.52$ & $1.91 \pm 1.05$ & $1.69 \pm 0.46$ & $1.24 \pm 0.09$ & $0.08 \pm 0.28$ & 270 \\
\hline
\end{tabular}


Table SI2. Amino acid yields for the four set of experiments after 31 days of hydrothermal reactions at $150^{\circ} \mathrm{C}, \mathrm{pH} 10$ and the relative abundance order of the 4 main amino acids, gly: glycine, ala: alanine, $\beta$-ala: $\beta$-alanine, $\gamma$-ABA: $\gamma$-aminobutyric acid.

\begin{tabular}{|c|c|c|}
\hline Initial Composition & Percent yield /HMT after 31 & Amino acid formed \\
\hline HMT & days, $150^{\circ} \mathrm{C}$ & gly $>>$ ala $>\beta$-ala $>\gamma$-ABA \\
\hline HMT+CA & 0.09 & gly $>>\beta$-ala $>$ ala $>\gamma$-ABA \\
\hline HMT+Al-rich & 0.06 & gly $>>\gamma$-ABA $>$ ala $>\beta$-ala \\
smectite & & gly $>>\gamma$-ABA $>\beta$-ala $>$ ala \\
\hline HMT+Fe-rich & 0.038 & \\
\hline smectite & & \\
\end{tabular}




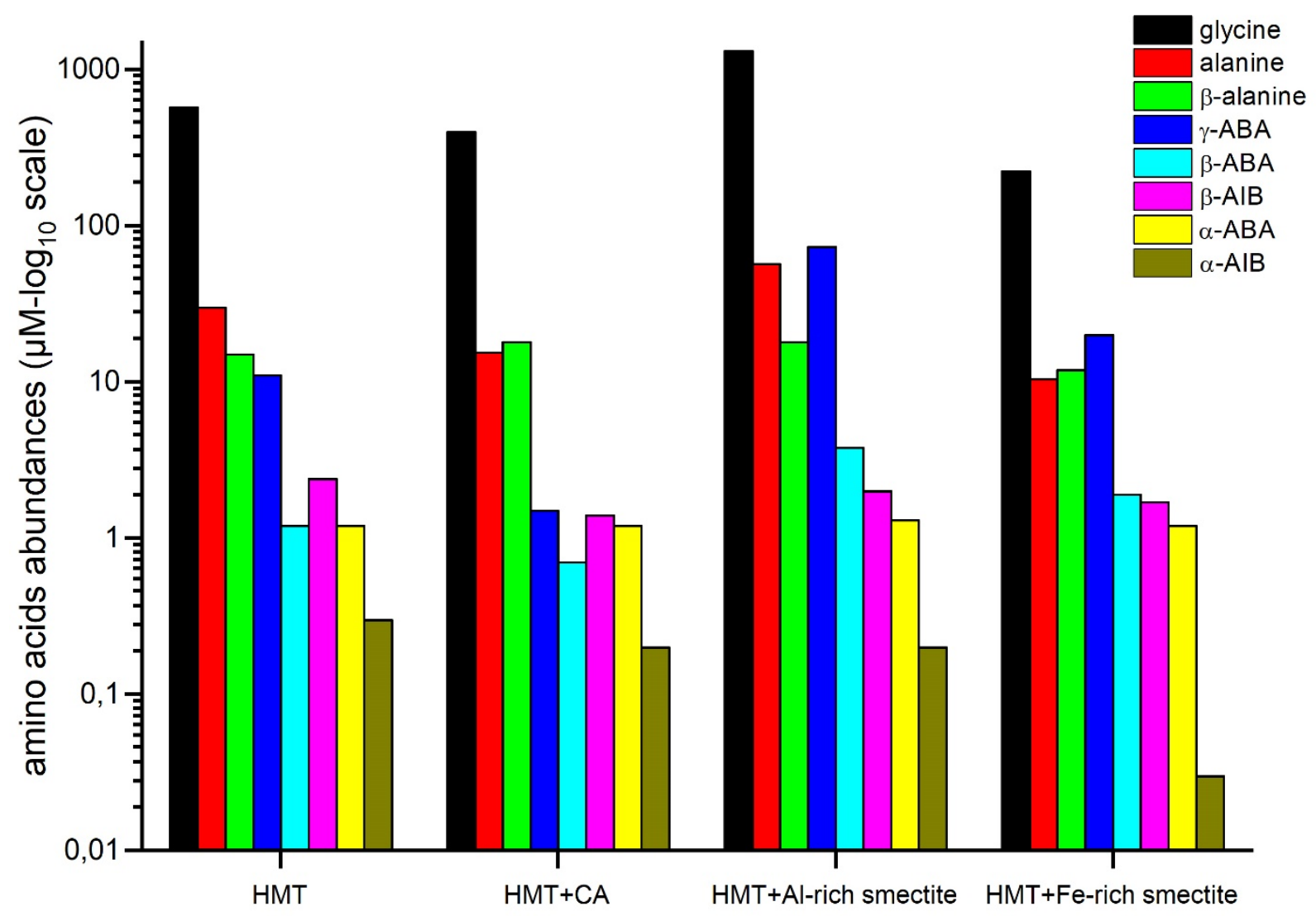

Figure SI2. Bar graph of the nature and distribution of the amino acids formed in function of the experiments from HMT-only, HMT+CA, HMT+Al-rich smectite and HMT+Fe-rich smectite after 31 day long hydrothermal alteration experiments at $150^{\circ} \mathrm{C}$. Scale are in $\log _{10}$ to increase the visibility of the low abundant compounds. 
a
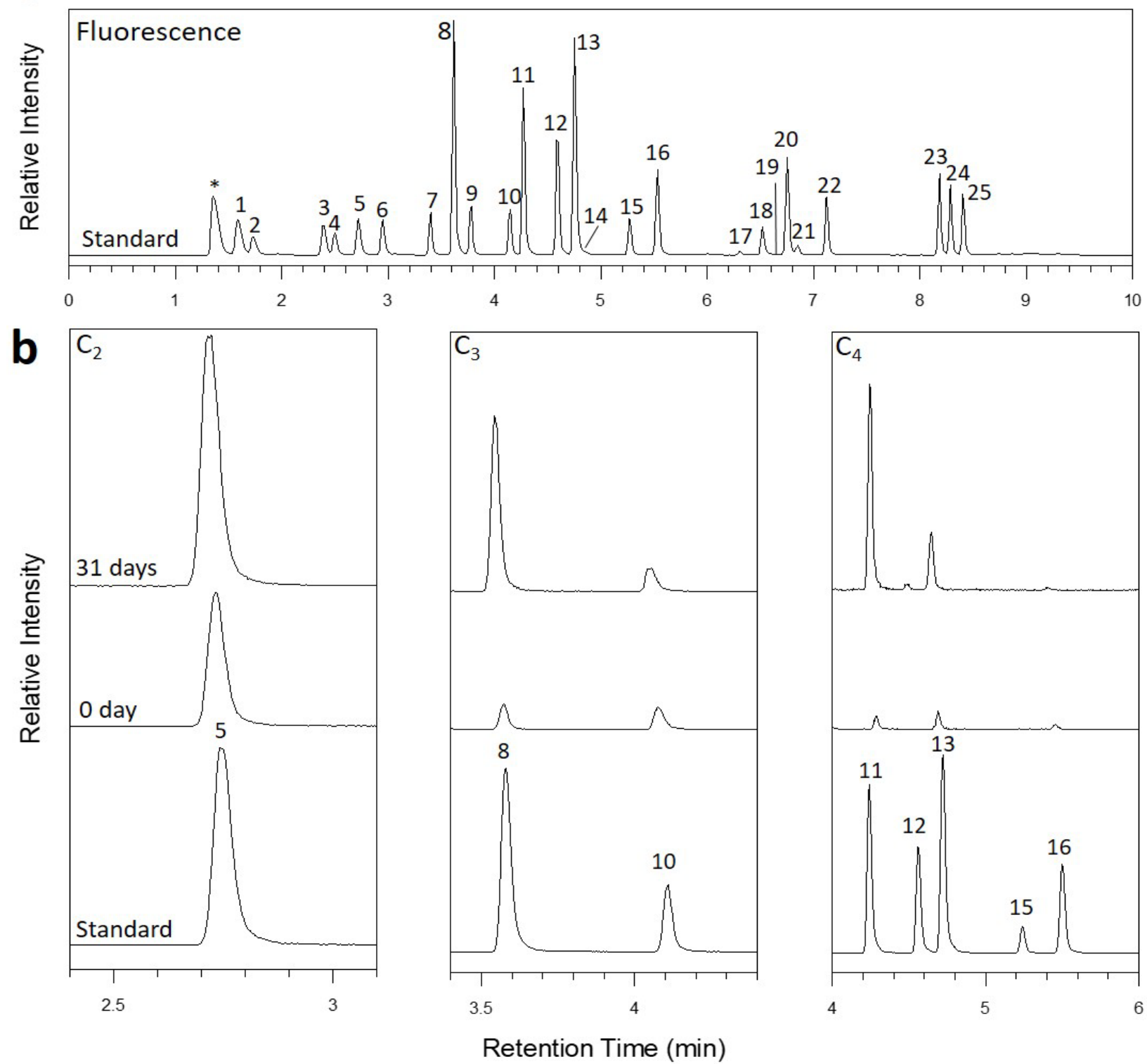

Figure SI3. Fluorescence chromatogram of the standard amino acids analyzed in this study (a) and zoom in the amino acids formed in the experiment with HMT-only after 31 days of hydrothermal alteration compared to the 0 day sample (unheated) (b). Scale has been maximized for clarity. Number referred to amino acid reported in table SI3 with their actual abundance. The * is a derivatization side product in the standard. Identification of the compounds is listed in Table SI3. 
Table SI3. List of amino acid and their abundance in $\mu \mathrm{M}$ analyzed in the HMT-only sample after 0, 2, 7, and 31 days of hydrothermal alteration. Peaks refer to the numbers in Figure SI2.

\begin{tabular}{|c|c|c|c|c|c|}
\hline Analyte & Peak & 0 day & 2 days & 7 days & 31 days \\
\hline Ammonia & 1 & & & & \\
\hline Histidine & 2 & $<0.1$ & $<0.1$ & $<0.1$ & $<0.1$ \\
\hline Serine & 3 & $<0.1$ & $<0.1$ & $<0.1$ & $<0.1$ \\
\hline Arginine & 4 & $<0.1$ & $<0.1$ & $<0.1$ & $<0.1$ \\
\hline Glycine & 5 & $0.4 \pm 0.1$ & $7.7 \pm 0.3$ & $\begin{array}{l}190.6 \pm \\
5.1\end{array}$ & $\begin{array}{l}573.4 \\
15.2\end{array}$ \\
\hline Aspartic acid & 6 & $<0.1$ & $<0.1$ & $<0.1$ & $<0.1$ \\
\hline Glutamic acid & 7 & $<0.1$ & $<0.1$ & $<0.1$ & $<0.1$ \\
\hline$\beta$-alanine & 8 & $<0.1$ & $\begin{array}{rr}1.0 \quad \pm \\
0.03\end{array}$ & $7.9 \pm 0.2$ & $15.4 \pm 0.4$ \\
\hline Threonine & 9 & $<0.1$ & $<0.1$ & $<0.1$ & $<0.1$ \\
\hline Alanine & 10 & $<0.1$ & $<0.1$ & $7.0 \pm 0.9$ & $30.3 \pm 2.1$ \\
\hline$\gamma$-amino butyric acid ( $\gamma$-ABA) & 11 & $<0.1$ & $<0.1$ & $1.2 \pm 0.2$ & $11.0 \pm 2.9$ \\
\hline
\end{tabular}




\begin{tabular}{|c|c|c|c|c|c|}
\hline $\begin{array}{l}\text { DL- } \beta \text {-aminobutyric acid (DL- } \beta \text { - } \\
\text { ABA) }\end{array}$ & 12 & $<0.1$ & $<0.1$ & $\begin{array}{rr}0.6 \quad \pm \\
0.03\end{array}$ & $1.2 \pm 1.1$ \\
\hline $\begin{array}{l}\text { DL- } \beta \text {-aminoisobutyric acid (DL- } \\
\beta \text {-AIB) }\end{array}$ & 13 & $<0.1$ & $<0.1$ & $0.9 \pm 0.2$ & $2.4 \pm 0.8$ \\
\hline Proline & 14 & $<0.1$ & $<0.1$ & $<0.1$ & $<0.1$ \\
\hline$\alpha$-aminoisobutyric acid $(\alpha-\mathrm{ABA})$ & 15 & $<0.1$ & $<0.1$ & $<0.1$ & $1.2 \pm 0.3$ \\
\hline$\alpha$-aminoisobutyric acid ( $\alpha$-AIB) & 16 & $<0.1$ & $<0.1$ & $<0.1$ & $0.3 \pm 0.2$ \\
\hline Cysteine & 17 & $<0.1$ & $<0.1$ & $<0.1$ & $<0.1$ \\
\hline Lysine & 18 & $<0.1$ & $<0.1$ & $<0.1$ & $<0.1$ \\
\hline Tyrosine & 19 & $<0.1$ & $<0.1$ & $<0.1$ & $<0.1$ \\
\hline$\varepsilon$-aminocaproic acid ( $\varepsilon$-ACA) & 20 & $<0.1$ & $<0.1$ & $<0.1$ & $<0.1$ \\
\hline Methionine & 21 & $<0.1$ & $<0.1$ & $<0.1$ & $<0.1$ \\
\hline Valine & 22 & $<0.1$ & $<0.1$ & $<0.1$ & $<0.1$ \\
\hline Leucine & 23 & $<0.1$ & $<0.1$ & $<0.1$ & $<0.1$ \\
\hline Isoleucine & 24 & $<0.1$ & $<0.1$ & $<0.1$ & $<0.1$ \\
\hline Phenylalanine & 25 & $<0.1$ & $<0.1$ & $<0.1$ & $<0.1$ \\
\hline
\end{tabular}




\section{Supplementary text}

\section{Amides in the HMT+CA mixtures}

\section{Analysis by GC-MS}

Compounds soluble in DCM were analyzed without derivatization, using an Agilent Technologies 6890N gas chromatograph coupled with an Agilent Technologies 5973 network mass spectrometer operated at the Laboratory Milieux Environnementaux, Transferts et Interactions dans les Hydrosystèmes et les Sols (METIS) in Paris, France. Description of the device is already described elsewhere $(34,35)$. The GC was equipped with a RTX-5Si/MS (30 m × $0.25 \mathrm{~mm}, 0.5$ $\mu \mathrm{m}$ film) capillary column coated with chemically bound Restek (low-polarity phase, suitable for semi-volatile, hydrocarbon, amine, phenol compounds). The injection temperature was $280{ }^{\circ} \mathrm{C}$ in spitless mode and the GC oven programmed from $50{ }^{\circ} \mathrm{C}$ to $320^{\circ} \mathrm{C}$, ramping at $4{ }^{\circ} \mathrm{C} \mathrm{min}^{-1}$, and using He as gas carrier. The solvent delay was as short as possible (3 minutes). The mass spectrometer was operated at an electron energy of $70 \mathrm{eV}$, an ion source temperature of $220^{\circ} \mathrm{C}$ and a scanning conditions from 35 to $700 \mathrm{amu}$ at $2.24 \mathrm{scan} \mathrm{s}^{-1}$. Products were identified using the NIST database. Data processing was done using the OpenChrom software, namely subtraction of the solvent background (DCM) taken at 3.8 min followed by an automatic peak detection using the MSD first derivative process (signal to noise ratios, $\mathrm{S} / \mathrm{N}>3$ ).

\section{Results}

In the presence of carboxylic acids with HMT in the starting solution, additional organic compounds are observed with the ones already observed in the HMT-only experiments (34). These compounds appear between 5 and 11 minutes on the chromatograms and are assigned to amide 
compounds, namely formamide, acetamide, and propanamide with N-methyl alkylation (Fig. SI4 and SI5, table SI4). The formation of some of these compounds may have inhibited the formation of other organic products, such as the one at $6.18,6.6$ or $7.8 \mathrm{~min}$; which are not observed in the chromatogram. These compounds are N-heterocycles such as imidazoline, or pyrazoline with alkyl substitution, as discussed in Table 1 of Reference (34). 


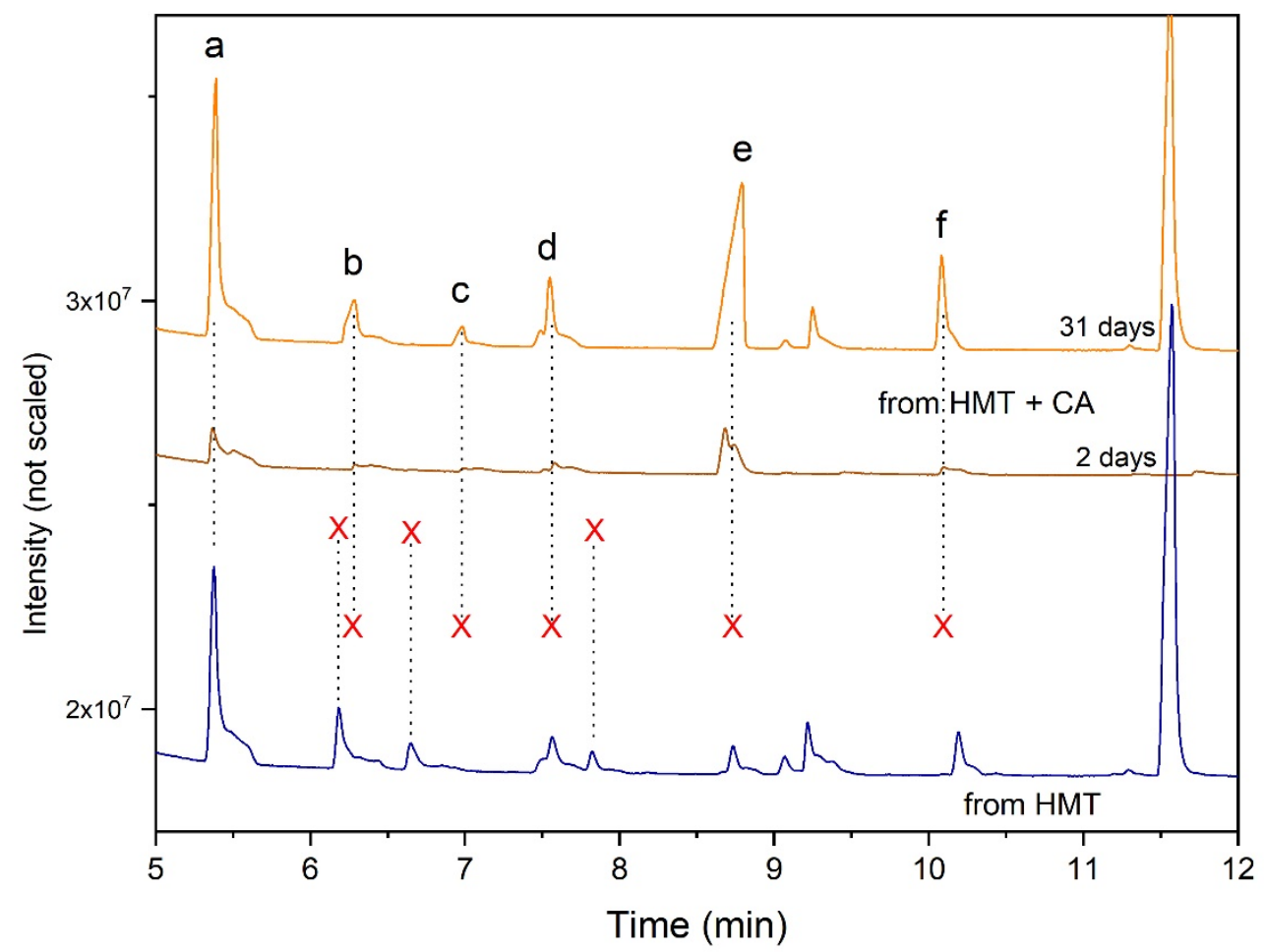

Figure SI4. GC-MS chromatograms of the DCM extract from the HMT+CA samples after 2 and 31 days of hydrothermal reaction, compared to the chromatogram from HMT alone after 31 days hydrothermal reaction (34). The lines ending by a red cross indicate the peaks present in the HMT+CA mixtures but not in the HMT-only mixture and reciprocally. 
Table SI4. Amides identified by GC-MS after 31 day long of hydrothermal experiments from the starting HMT+CA (0.7 M). See Figure SI5 for the total mass spectrum of each amides. See Vinogradoff et al. (34) for identification of the rest of peaks in the chromatogram.

\begin{tabular}{|c|c|c|c|c|c|}
\hline \multirow[t]{2}{*}{$\begin{array}{r}\text { Figure } \\
\text { SI3 label }\end{array}$} & \multirow[t]{2}{*}{$\begin{array}{l}\text { Retention } \\
\text { time }\end{array}$} & \multirow[t]{2}{*}[\mathrm{M}+]{} & \multicolumn{2}{|l|}{ Designation } & \multirow[t]{2}{*}{ Structures } \\
\hline & & & name & formula & \\
\hline $\mathrm{a}$ & 5.37 & 73 & $\begin{array}{l}\text { N,N-dimethyl } \\
\text { formamide }\end{array}$ & $\mathrm{C}_{3} \mathrm{H}_{7} \mathrm{NO}$ & \\
\hline $\mathrm{b}$ & 6.27 & 73 & N-methyl acetamide & $\mathrm{C}_{3} \mathrm{H}_{7} \mathrm{NO}$ & \\
\hline C & 6.97 & 73 & propanamide & $\mathrm{C}_{3} \mathrm{H}_{7} \mathrm{NO}$ & \\
\hline $\mathrm{d}$ & 7.55 & 87 & $\begin{array}{l}\text { N,N-dimethyl } \\
\text { acetamide }\end{array}$ & $\mathrm{C}_{4} \mathrm{H}_{9} \mathrm{NO}$ & \\
\hline e & 8.75 & 87 & N-methyl propanamide & $\mathrm{C}_{4} \mathrm{H}_{9} \mathrm{NO}$ & \\
\hline
\end{tabular}




\begin{tabular}{|l|l|l|l|l|l|}
\hline $\mathrm{f}$ & 10.07 & 101 & N,N-dimethyl & $\mathrm{C}_{5} \mathrm{H}_{11} \mathrm{NO}$ \\
\hline
\end{tabular}
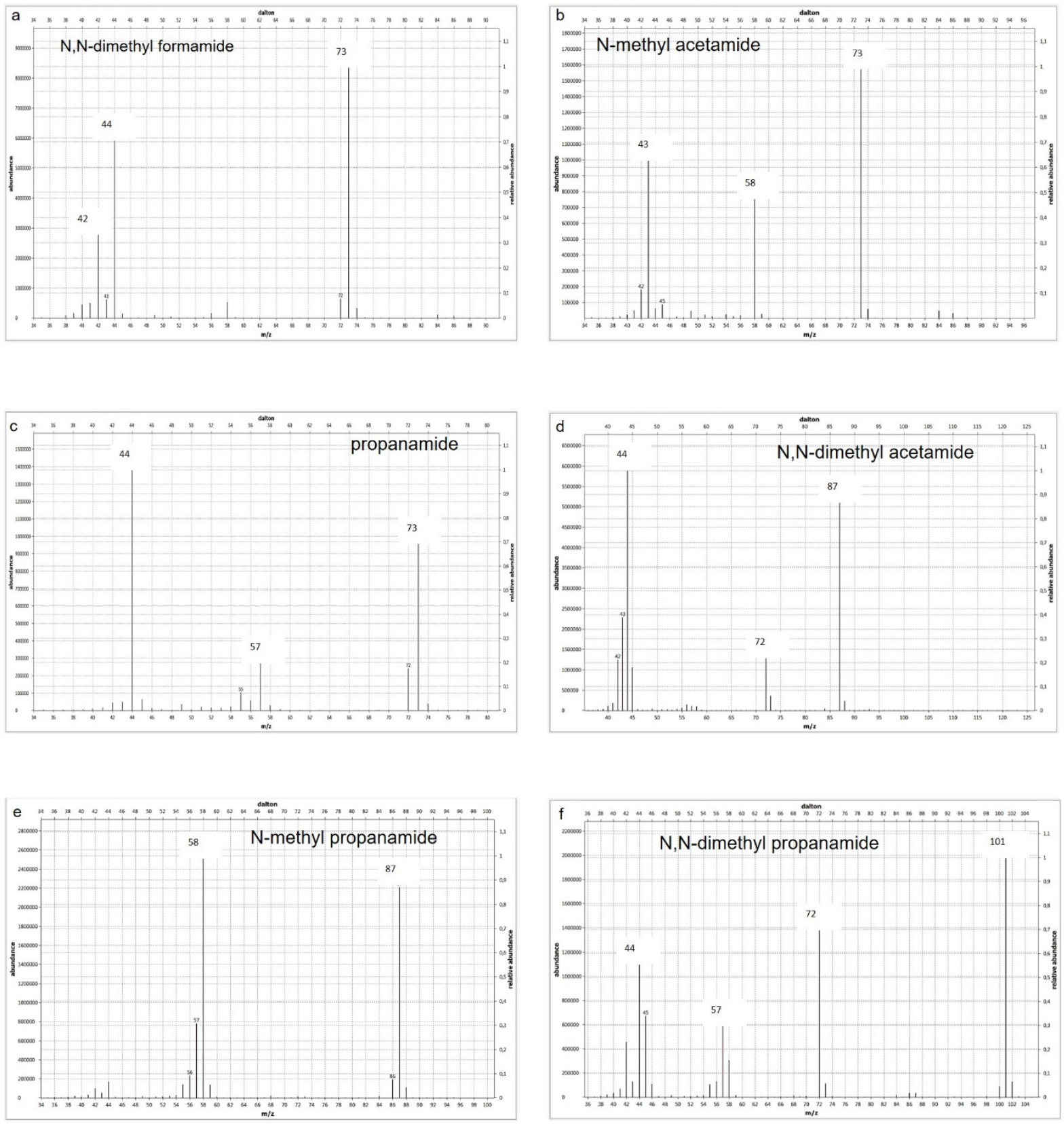

Figure SI5 Mass spectra of the compounds a to f labelled in figure SI4 corresponding to amides reported in Table SI4. $\mathrm{m} / \mathrm{z}$ of the major peaks are reported for clarity. 